\title{
On the Origin of Bipolar Planetary Nebulae
}

\author{
Shigeki Miyaji and Daigo Saito \\ Department of Physics, Graduate school of Science and Technology, Chiba University, \\ Chiba 2638522, Japan \\ email: miyaji@astro.s.chiba-u.ac.jp
}

\begin{abstract}
We present a numerical simulation of the formation of a bipolar planetary nebula from a single star. At the late stage of AGB stars, surface convection and/or convection of shell burning would create differential rotation between the core and the envelope of such stars. If the core has a magnetic field threading the envelope, this differential rotation twists magnetic fields. Then like a cosmic jet model of accretion disks, such a twist of magnetic fields piles up a tower of magnetic fields atop the pole of the progenitor core and creates jets.
\end{abstract}

Keywords. convection, instabilities, MHD, plasma, planetary nebulae: general, stars: post-AGB

\section{Introduction and Summary}

We simulate jet formation from a single star consisting of a rotating magnetized core and its gas pressure dominated envelope at rest. Since our single star model creates jets similar to those made by binary accretion disk models (e.g., Kato, Hayashi \& Matsumoto 2004 and Frank 2006), we need higher resolution observations of bipolar planetary nebulae at the origin of jets and at the mother star position in order to distinguish whether or not the mother star is in a binary system.

Our model differs from the accretion disk jet model mainly in the origin of jets. In our case, the jet originates from the polar cusp of the magnetosphere by a Sausage instability. In the accretion disk case, a surface avalanche of the disk creates mass evaporation from the disk and the resulting plasma moves along magnetic field lines. In both cases, a tower of piled up azimuthal components of magnetic fields dominates the jet, so that it is self-collimated because of its magnetic tension (see e.g., Garcia-Segura, Alberto, \& Jose 2005). In the case of a 3D simulation, it shows kink instability when $v_{z}$ exceeds the Alfven velocity (Kuwabara, Shibata, Kudoh et al. 2005).

\section{Models, Code, and Results}

The core is assumed to be a $1 \mathrm{M}_{\odot}$ white dwarf with core radius $R_{c}$. We also assume that the core is a rigid rotator with 0.1 of the Kepler velocity $v_{0}$ at its surface. The envelope at rest has a polytropic structure with $N=3$ and its bottom density is set to be $1 \mathrm{~g} / \mathrm{cm}^{-3}$. The core has a dipole magnetic field which threads the envelope and its strength is a free parameter in our models.

We used a 2-dimensional cylindrical MHD code with Modified Lax-Wendroff scheme from CANS (URL: http://www.astro.phys.s.chiba-u.ac.jp/netlab/, Coordinated Astronomical Numerical Software). The basic equations employed are as usual MHD equations with artificial viscosity. We assume an axisymmetric simulation region with $(r, z)=$ $(300,300)$ grids $=\left(62 \mathrm{R}_{\mathrm{c}}, 62 \mathrm{R}_{\mathrm{c}}\right)$. The inner part of the simulation region $\left(r, z<8.9 R_{c}\right)$ has a high constant resolution; the resolution becomes gradually coarser outwards. As a 


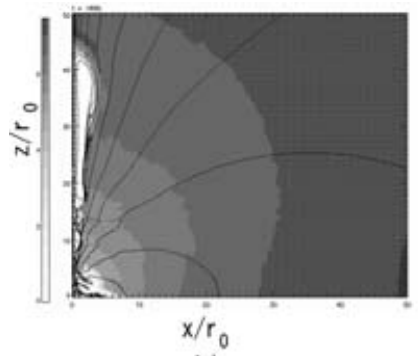

(a)

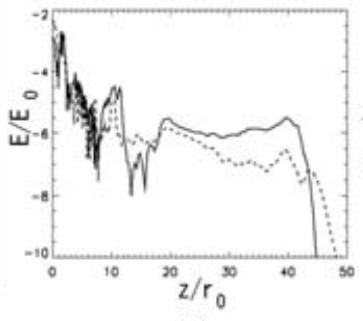

(b)

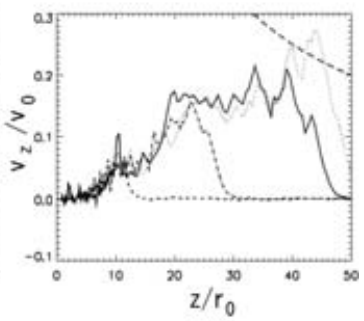

(c)

Figure 1. (a) Contours of Plasma $\beta$ and Magnetic Fields at the Time of 27 Core Rotations of Model 2. Black lines show magnetic field lines. (b) Energy Flux Profiles at 27 core rotations at $x=0.5 R_{c}$. Thick line shows Poynting flux and dashed line shows kinetic energy flux in units of $E_{0}=1 / 2 v_{0}{ }^{2}$. (c) Vertical velocity profiles at $x=0.5 R_{c}$ at 22 (dashed), 24.5 (dash-dotted), 27 (solid), and 27.5 (dotted) core rotation. Long dashed line shows escape velocity.

Table 1. Parameters at the Time of Jet Formation

\begin{tabular}{l|cccccc}
\hline Model & 1 & 2 & 3 & 4 & 5 & 6 \\
\hline $\log B(\mathrm{G})$ & 6.00 & 7.00 & 7.25 & 7.50 & 7.75 & 8.00 \\
$R_{m} / R_{c}$ & 2.0 & 4.7 & 6.5 & 8.5 & 12.5 & 14.0 \\
Jet Width $/ R_{c}$ & 0.5 & 0.8 & 1.2 & 1.5 & 1.5 & 3.0 \\
$T_{\text {core rotation }}$ & 31 & 19 & 25 & 28 & 32 & 32 \\
\hline
\end{tabular}

boundary condition, we assume mirror boundaries at $r$ and $z$ axes and fixed boundary with a buffer zone at the outer boundary and also at the surface of the core.

In all models, we found formation of jets and equatorial plane flows (Table 1). With weak magnetic fields, differential rotation makes the magnetic field extend in the azimuthal direction. Then the created azimuthal component of magnetic field $\left(B_{\phi}\right)$ becomes dominant and piles up like a magnetic tower jet model (Kato et al. 2004). With stronger initial magnetic field, the equatorial flow becomes dominant because strong magnetic tension enhances the centrifugal force through angular momentum transport from the core to the envelope. However, when the equatorial plane flow reaches a certain magnitude it turns to be a strong anchor of the magnetic fields. Then, in a similar way as with accretion disk models, such an anchor could power up already existing piled up magnetic fields.

When the rotation proceeds, the plasma $\beta$ (Gas Pressure/ Magnetic Pressure) becomes as low as and then saturated around 10. We call this low $\beta$ region a "magnetosphere" with mean radius $R_{m}$. At the outer equator of the magnetosphere, the magnetic tension force makes the plasma corotate, and an equatorial plane flow is formed. At the cusp atop the magnetic pole, the high azimuthal field forces the Sausage instability to grow. As a result, a Poynting flux dominated knot is formed and its vertical velocity exceeds the escape velocity and becomes a "jet" (see figure 1(b) and (c)).

We (especially D. S.) would like to acknowledge useful comments and encouragement by Dr. T. Hanawa and Dr. R. Matsumoto.

\section{References}

Frank, A. 2006, these Proceedings

Garcia-Segura, G., Alberto, L. J., \& Jose, F. 2005, ApJ 618, 919

Kato, Y., Hayashi, M. R., \& Matsumoto, R. 2004, ApJ 600, 338

Kuwabara, T., Shibata, K., Kudoh, T. \& Matsumoto, R. 2005, ApJ 621, 921 